\title{
Improved Stroke Care in a Primary Stroke Centre Using Al-Decision Support
}

\author{
Bence Gunda ${ }^{a}$ Ain Neuhaus ${ }^{b} \quad$ Ildikó Sipos $^{a}$ Rita Stang ${ }^{a}$ Péter Pál Böjti ${ }^{a}$

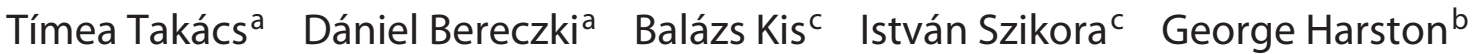 \\ ${ }^{a}$ Department of Neurology, Semmelweis University, Budapest, Hungary; ${ }^{b}$ Oxford University Hospitals NHS \\ Foundation Trust, Oxford, UK; ' National Institute of Mental Health, Neurology and Neurosurgery, Budapest, \\ Hungary
}

\section{Keywords}

Stroke · Thrombolysis · Thrombectomy · Machine learning · e-ASPECTS

\begin{abstract}
Background: Patient selection for reperfusion therapies requires significant expertise in neuroimaging. Increasingly, machine learning-based analysis is used for faster and standardized patient selection. However, there is little information on how such software influences real-world patient management. Aims: We evaluated changes in thrombolysis and thrombectomy delivery following implementation of automated analysis at a high volume primary stroke centre. Methods: We retrospectively collected data on consecutive stroke patients admitted to a large university stroke centre from two identical 7-month periods in 2017 and 2018 between which the e-Stroke Suite (Brainomix, Oxford, UK) was implemented to analyse non-contrast CT and CT angiography results. Delivery of stroke care was otherwise unchanged. Patients were transferred to a hub for thrombectomy. We collected the number of patients receiving intravenous thrombolysis and/or thrombectomy, the time to treatment; and outcome at 90 days for thrombectomy. Results: 399 patients from 2017 and 398 from 2018 were included in the study. From 2017 to 2018, thrombolysis rates increased from $11.5 \%$ to $18.1 \%$ with a similar trend for thrombectomy $(2.8-$
\end{abstract}

4.8\%). There was a trend towards shorter door-to-needle times (44-42 $\mathrm{min}$ ) and CT-to-groin puncture times (174-145 min). There was a non-significant trend towards improved outcomes with thrombectomy. Qualitatively, physician feedback suggested that e-Stroke Suite increased decisionmaking confidence and improved patient flow. Conclusions: Use of artificial intelligence decision support in a hyperacute stroke pathway facilitates decision-making and can improve rate and time of reperfusion therapies in a huband-spoke system of care.

(C) 2022 The Author(s)

Published by S. Karger AG, Basel

\section{Introduction}

Reperfusion therapy is central to modern acute ischaemic stroke management. The most widely available form of this is thrombolysis with intravenous tissue plasminogen activator (tPA) [1]. More recently, mechanical thrombectomy (MT) has revolutionized treatment of large vessel occlusion (LVO) ischemic stroke, with a numberneeded-to-treat to improve functional outcome as low as 2.6 [2], making it both clinically highly efficacious and cost-effective [3].

Despite evidence for their effectiveness, only a minority of stroke patients receive reperfusion therapy [4], due to clinical contraindications, risk-benefit balance assess-
C 2022 The Author(s).

Published by S. Karger AG, Basel

This is an Open Access article licensed under the Creative Commons Attribution-NonCommercial-4.0 International License (CC BY-NC) (http://www.karger.com/Services/OpenAccessLicense), applicable to the online version of the article only. Usage and distribution for commercial purposes requires written permission.
Correspondence to:

Bence Gunda, bence.gunda@gmail.com 
ment, and delayed presentation beyond the evidencebased treatment window. The problem is exacerbated by delays in appropriate triage and imaging, required to determine patient eligibility. Stroke treatment is becoming increasingly personalised: appropriate imaging-guided patient selection has enabled treatment up to $24 \mathrm{~h}$ from onset for MT [5, 6] and up to $9 \mathrm{~h}$ for thrombolysis [7-9].

However, such analyses require time and significant neuroradiological expertise. As such, there is significant interest in optimizing image analysis protocols to reduce treatment times and more accurately select patients. One increasingly popular method is automated image analysis, most notably machine learning algorithms that characterize ischemic regions from non-contrast CT and identify occlusions on CT angiography. e-Stroke is a widely available CE-marked software package that incorporates analysis tools for infarct size on non-contrast CT (e-ASPECTS) and for LVO and collateral flow on CT angiography, and provides a useful decision-making aid for imaging-guided selection. e-ASPECTS has been validated as being non-inferior to experienced neuroradiologists for identifying ASPECTS on CT [10]. However, there is little data how automated analysis alters management in a real-world setting. In this study, we characterized two periods in consecutive years, in between which e-Stroke software was deployed at a primary stroke centre in a hub-and-spoke system of care, to determine its impact on reperfusion therapy uptake and speed, and whether this altered outcomes in MT.

\section{Methods}

Consecutive patients admitted to the Department of Neurology, Semmelweis University (Budapest, Hungary) between May and December in 2017 and 2018 with acute ischemic stroke were retrospectively analysed. Patients were selected for reperfusion treatment based on established international and local guidelines at that time. Briefly, they were eligible for thrombolysis if they presented within $4.5 \mathrm{~h}$ of stroke onset, had no evidence of haemorrhage or other clinical contraindications, and did not have definite hypodensity exceeding 2/3 of MCA territory. Patients were considered eligible for thrombectomy if they had no evidence of haemorrhage or other clinical contraindications and had an ASPECTS score $>5$ and occlusion of the intracranial internal carotid, middle cerebral, or basilar artery. Data collected included age, sex, timeto-treatment (door-to-needle for thrombolysis, first scan-to-groin puncture for thrombectomy), National Institutes of Health Stroke Scale (NIHSS) on admission. Modified Rankin Scale (mRS) at 90 days was assessed in MT treated patients by trained personnel via telephone interviews as standard practice.

Non-contrast CT scans were obtained using a 16-slice scanner (Philips, Amsterdam, The Netherlands), with slice thickness set at $2 \mathrm{~mm}$ for NCCT and $1 \mathrm{~mm}$ for CTA. e-ASPECTS and e-CTA anal-

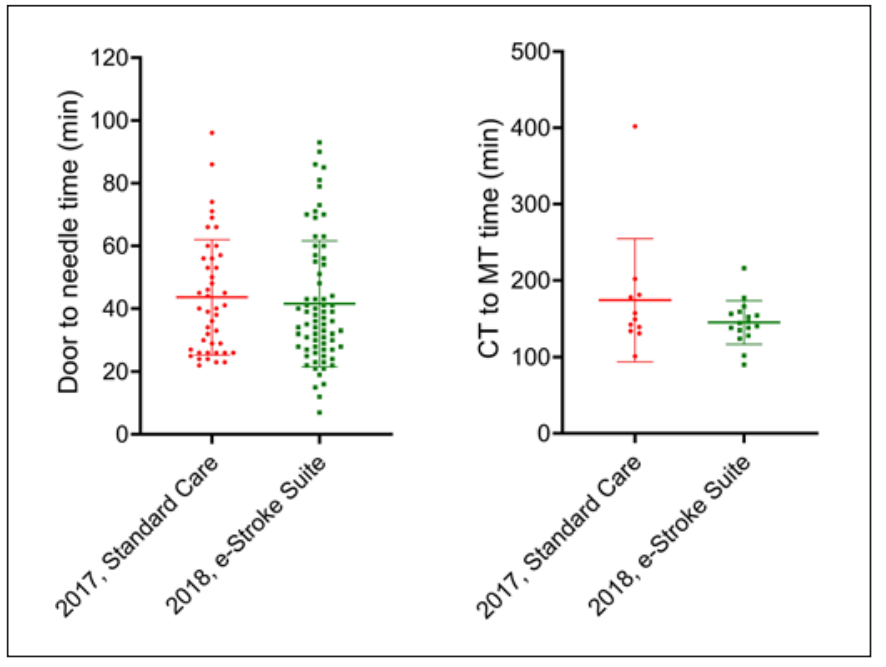

Fig. 1. Treatment times for patients in consecutive years for thrombolysis (left) and MT (right).

ysis was performed as previously described [10]. DICOM images from non-contrast CT and CT angiography were pre-processed and corrected for positional transformations. e-ASPECTS then automatically segmented regions of the MCA territory and characterized the tissue as ischaemic or normal-appearing; outputs included ASPECTS and acute ischemic volume. e-CTA outputs included LVO location, ratio of collateral flow compared to the contralateral side, and a collateral score (with 0 as no flow and 3 as complete collateral blood supply) [11].

Changes in the number of patients receiving reperfusion therapy were characterized using $\chi^{2}$ analysis. Door-to-treatment times were compared using a Student's $t$ test. Outcome analyses were performed for dichotomized mRS (with $0-2$ as good outcome, $0-1$ as excellent outcome) using $\chi^{2}$ analysis and for mRS shift using Wilcoxon rank sum test. Data are presented as mean with standard deviation for continuous variables, and median with interquartile range for ordinal variables including NIHSS and mRS. Statistical analyses were performed in $\mathrm{R}$.

\section{Results}

In a 7-month period during 2017, 399 patients were admitted to the Department of Neurology at Semmelweis University with acute ischemic stroke. 46 patients (11.5\%) were treated with IV-tPA, with a mean age of $67.6 \pm 13.3$ years, $45.7 \%$ male, and a median NIHSS of $8(5-13)$. The mean door-to-needle time was $44 \pm 18 \mathrm{~min}$ (based on data from 44 patients; in 2 patients thrombolysis time was not documented, however other time stamped data suggest they fell outside $30 \mathrm{~min}$ therefore unlikely to change statistics). In an equivalent 7 -month period in 2018, following e-Stroke implementation, 398 stroke patients were 
Fig. 2. Thrombolysis treatment rates over 2-month periods during 2017 and 2018.

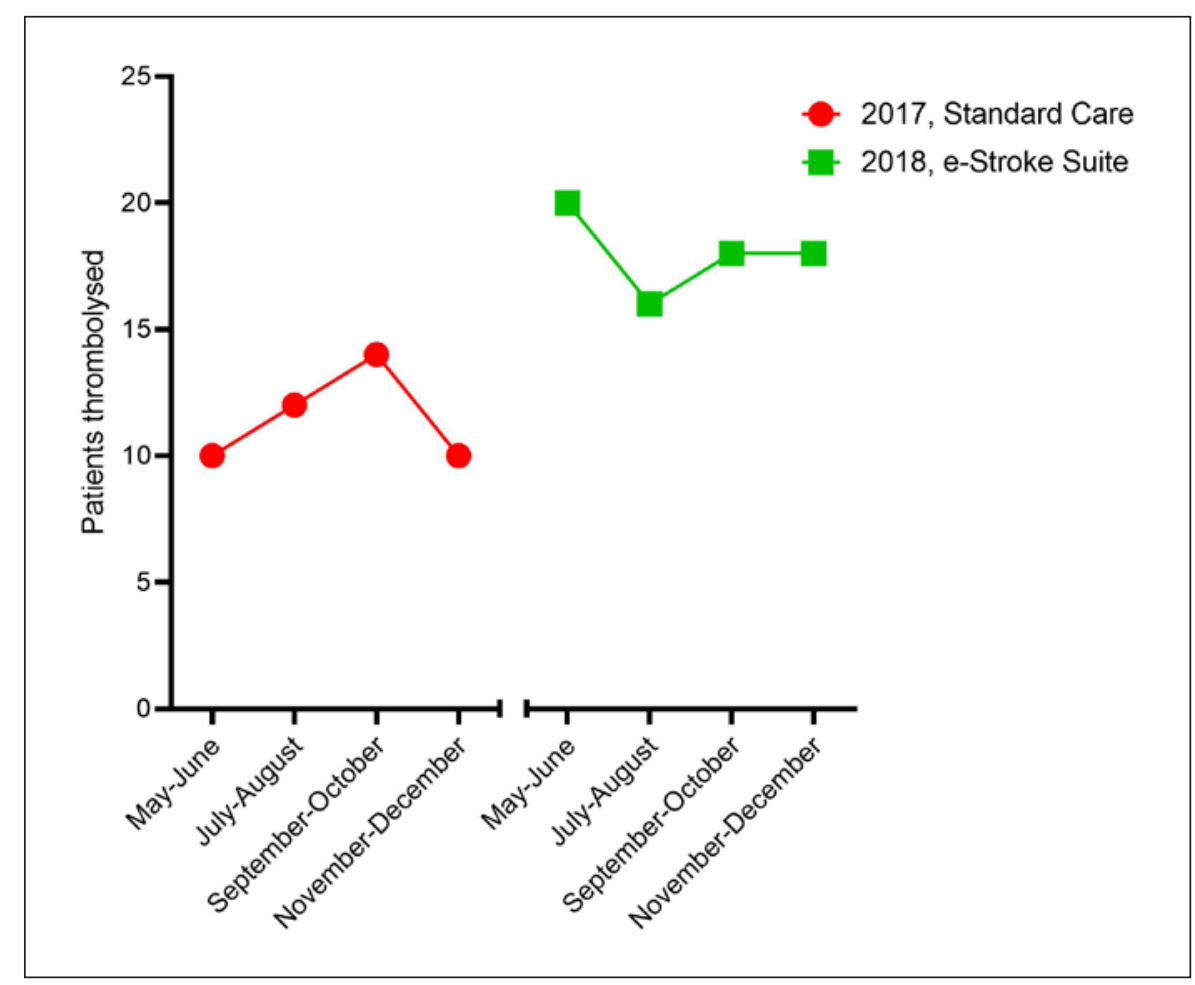

admitted. 72 patients (18.1\%) were treated with IV-tPA, with a mean age of $65.1 \pm 13.5$ years, $55.6 \%$ male and a median NIHSS of $6(3-10.25)$. This represented a $56.9 \%$ increase in the number of patients thrombolyzed compared to 2017 ( $p=0.009$ ). The mean door-to-needle time was $42 \pm 20 \mathrm{~min}$, a statistically non-significant decrease of $4.5 \%$ compared to 2017 ( $p=0.57$, Fig. 1 ). There was no apparent trend in treatment rates within each year period (Fig. 2).

In 2017,11 patients $(2.8 \%)$ received MT, with a mean age of $55.8 \pm 18.1$ years, $54.5 \%$ male, and a median NIHSS of 15 (13.5-18.5) on admission. The mean CT-to-groin puncture time was $174 \pm 80.5 \mathrm{~min}$. In 2018, this increased to 19 patients $(4.8 \%)$, with a mean age of $62.3 \pm 15.3$ years, $52.6 \%$ male and a median NIHSS of $13(10-15.5)$ on admission. This represented a statistically non-significant rise of $72.7 \%$ in the number of patients receiving thrombectomy $(p=0.13)$. The mean CT-to-groin puncture time was $145 \pm 28 \mathrm{~min}$ (based on data from 17 patients), a decrease of $16.7 \%$ compared to 2017 ( $p=0.29)$.

Outcome data at 90 days were available for the MT cohort in 10 patients from 2017 and 18 patients from 2018 (Fig. 3). Good functional outcome (mRS 0-2) was achieved by 6 patients in 2017 and 11 patients in 2018 ( $p$ $=1$ ). Excellent outcome (mRS 0-1) was achieved by 2 pa- tients in 2017 and 7 patients in 2018 ( $p=0.55)$. When analysed for mRS shift, there was a trend towards improved outcomes in $2018(p=0.29)$.

\section{Discussion}

Our study describing a positive real-world impact of AI-decision support software on acute stroke management is in line with two recent reports on MT results in a similar hub-and-spoke system of care $[12,13]$. We noted a significant increase in thrombolysis rates and a trend towards increased transfer for MT. Treatment times and outcomes of those patients treated with MT also showed a trend towards improvement.

Exploratory qualitative non-structured oral feedback from clinicians using the e-Stroke Suite identified several factors that were thought to underlie the effects seen. Increased confidence and speed of image interpretation improved local decision-making. Image sharing capabilities via the e-Stroke platform between clinicians in a huband-spoke system was also felt to make multidisciplinary treatment decisions more rapid as there is no well-established patient data and image sharing pathway between our hospitals using different PACS systems.
Gunda/Neuhaus/Sipos/Stang/Böjti/ Takács/Bereczki/Kis/Szikora/Harston 


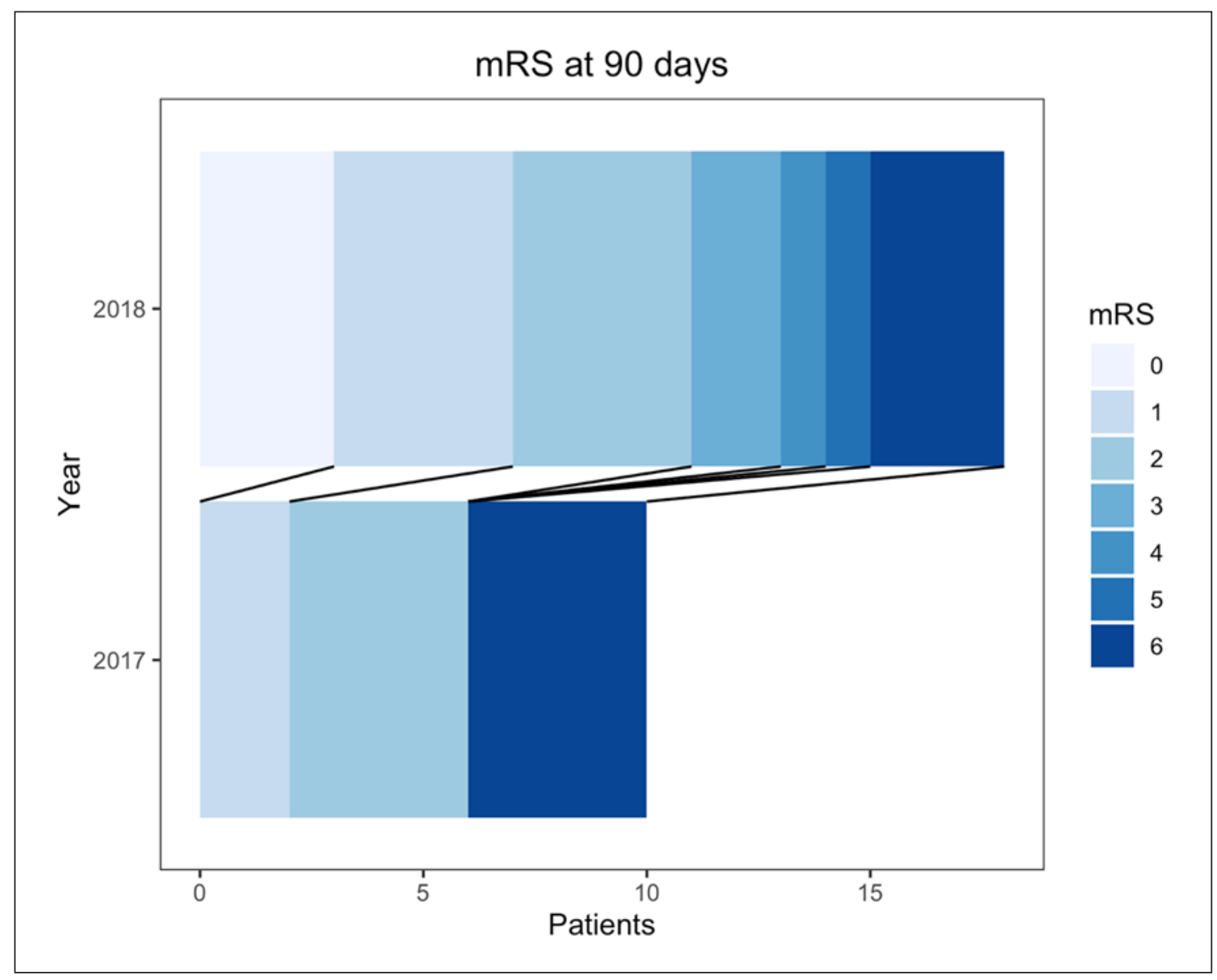

Fig. 3. mRS distributions at 90 days following stroke in the MT cohort.

This study is limited by its observational nature. Although no changes other than the introduction of the eStroke Suite were made to service delivery over the duration of the project, we cannot exclude other factors contributing to improved stroke care, such as increased public awareness of stroke and ongoing quality improvement at the department. However, exploratory analysis of trends over time did not show any change in treatment rates within the individual years for thrombolysis -a stable and well-established treatment. This analysis is based on a relatively small sample size and was underpowered to detect change in treatment times, rates of MT, or changes in clinical outcome. Future multi-centre studies with random allocation of decision support would provide more definitive results.

This study supports the principle that AI-powered image interpretation can support clinicians in making treatment decisions. The results support further evaluation of this technology routinely incorporated into stroke clinical care pathways.

\section{Statement of Ethics}

This retrospective review of patient data did not require ethical approval in accordance with local guidelines. Written informed consent from participants was not required in accordance with local guidelines.

\section{Conflict of Interest Statement}

Dr. Harston is the chief medical officer at Brainomix. The other authors declare no conflict of interest.

\section{Funding Sources} ESO). 


\section{Author Contributions}

Bence Gunda and George Harston contributed to the study conception and design. Material preparation, data collection, and analysis were performed by Bence Gunda, Ain Neuhaus, Ildikó Sipos, Rita Stang, Péter Böjti, Tímea Takács, and Balázs Kis. The first draft of the manuscript was written by Bence Gunda and Ain Neuhaus. George Harston, Dániel Bereczki, and István Szikora contributed significantly with intellectual guidance and comments. All authors read and approved the final manuscript.

\section{Data Availability Statement}

The data that support the findings of this study are available from the corresponding author on reasonable request.

\section{References}

1 Wardlaw JM, Murray V, Berge E, del Zoppo GJ. Thrombolysis for acute ischaemic stroke. Cochrane Database Syst Rev. 2014;2014(7): CD000213.

2 Goyal M, Menon BK, van Zwam WH, Dippel DW, Mitchell PJ, Demchuk AM, et al. Endovascular thrombectomy after large-vessel ischaemic stroke: a meta-analysis of individual patient data from five randomised trials. Lancet. 2016;387(10029):1723-31.

3 Lobotesis K, Veltkamp R, Carpenter IH, Claxton LM, Saver JL, Hodgson R. Cost-effectiveness of stent-retriever thrombectomy in combination with IV t-PA compared with IV t-PA alone for acute ischemic stroke in the UK. J Med Econ. 2016;19(8):785-94.

4 Donnan GA, Davis SM, Parsons MW, Ma H, Dewey HM, Howells DW. How to make better use of thrombolytic therapy in acute ischemic stroke. Nat Rev Neurol. 2011;7(7):400-9.

5 Albers GW, Marks MP, Kemp S, Christensen S, Tsai JP, Ortega-Gutierrez S, et al. Thrombectomy for stroke at 6-16 hours with selection by perfusion imaging. $\mathrm{N}$ Engl $\mathrm{J}$ Med. 2018;378(8):708-18.
6 Nogueira RG, Jadhav AP, Haussen DC, Bonafe A, Budzik RF, Bhuva P, et al. Thrombectomy 6-24 hours after stroke with a mismatch between deficit and infarct. N Eng J Med. 2018;378(1):11-21.

7 Campbell BCV, Ma H, Ringleb PA, Parsons MW, Churilov L, Bendszus M, et al. Extending thrombolysis to $4 \cdot 5-9 \mathrm{~h}$ and wake-up stroke using perfusion imaging: a systematic review and meta-analysis of individual patient data. Lancet. 2019;394(10193):139-47.

$8 \mathrm{Ma} \mathrm{H}$, Campbell BCV, Parsons MW, Churilov L, Levi CR, Hsu C, et al. Thrombolysis guided by perfusion imaging up to 9 hours after onset of stroke. N Engl J Med. 2019; 380(19):1795-803.

9 Berge E, Whiteley W, Audebert H, De Marchis GM, Fonseca AC, Padiglioni C, et al. European Stroke Organisation (ESO) guidelines on intravenous thrombolysis for acute ischaemic stroke. Eur Stroke J. 2021;6(1):I-LXII.
10 Nagel S, Sinha D, Day D, Reith W, Chapot R, Papanagiotou P, et al. e-ASPECTS software is non-inferior to neuroradiologists in applying the ASPECT score to computed tomography scans of acute ischemic stroke patients. Int J Stroke. 2017;12(6):615-22.

11 Tan IY, Demchuk AM, Hopyan J, Zhang L, Gladstone D, Wong K, et al. CT angiography clot burden score and collateral score: correlation with clinical and radiologic outcomes in acute middle cerebral artery infarct. AJNR Am J Neuroradiol. 2009;30(3):525-31.

12 Morey JR, Zhang X, Yaeger KA, Fiano E, Marayati NF, Kellner CP, et al. Real-world experience with artificial intelligence-based triage in transferred large vessel occlusion stroke patients. Cerebrovasc Dis. 2021;50(4):450-5.

13 Hassan AE, Ringheanu VM, Rabah RR, Preston L, Tekle WG, Qureshi AI. Early experience utilizing artificial intelligence shows significant reduction in transfer times and length of stay in a hub and spoke model. Interv Neuroradiol. 2020;26(5):615-22. 